\title{
Simplified method for cell-specific gene expression analysis in Caenorhabditis elegans
}

Takuma Sugi ${ }^{1,2, *}$ and Yasuko Ohtani ${ }^{1}$

${ }^{1}$ Institute for Integrated Cell-Material Sciences (WPI-iCeMS), Kyoto University, Yoshida-Honmachi, Sakyo-ku, Kyoto 606-8501, Japan

${ }^{2}$ PRESTO, Japanese Science and Technology Agency, 4-1-8 Honcho, Kawaguchi, Japan

*Corresponding author: Takuma Sugi (tsugi@icems.kyoto-u.ac.jp)

81-75-383-2164 (voice)

81-75-383-2541 (FAX)

Kyoto daigaku-Katsura A4 Room136, Nishikyo-ku, Kyoto 615-8510, Japan 


\begin{abstract}
In the neural circuit functional identities of individual neurons are mainly specified by their differential gene expression patterns. Unveiling functional roles of each neuron requires cell-specific interrogation of neural circuitry in the context of gene expressions. The mRNA tagging strategy in Caenorhabditis elegans is a powerful technique, in which cell-specific transcripts can be isolated by co-immunoprecipitating the complexes of mRNAs and epitope-tagged poly(A) binding protein (3× FLAG-PAB-1), expressed in target neurons. However, the conventional protocol requires laborious and time-consuming procedures; chromosomal integration of gene encoding 3× FLAG-PAB-1 and bleaching of obtained integrant animals for the isolation of huge amounts of synchronized animals. In this paper, we have presented a simplified methodology for cell-specific mRNA tagging analysis in $C$. elegans. We show that mRNA tagging was achieved using transgenic animals expressing $3 \times$ FLAG-PAB-1 as an extrachromosomal array under the control of the flp-18 promoter, without the chromosomal integration procedure. Furthermore, we successfully isolated cell-specific mRNAs from adult transgenic animals synchronously grown from eggs laid by gravid adults during a time window of 3 hours. This simplification facilitates the implementation of cell-specific gene expression analysis of C. elegans, which contributes to the understanding of neural circuitry at a cell-specific resolution.
\end{abstract}




\section{Introduction}

The mammalian nervous system is composed of a massive number of neurons and their synaptic connections. Functional identities of individual neurons are determined by their differential gene expression patterns, which enable brain functions ranging from neural differentiation to memory formation. Therefore, understanding functional roles of each neuron inevitably requires cell-specific interrogations of gene expression changes in the context of each brain function. However, this interrogation was hampered by the complexity of the mammalian nervous system.

The nematode Caenorhabditis elegans is a genetically tractable model animal with a compact nervous system consisting of only 302 neurons. The simplicity of C. elegans offers a unique opportunity to express a gene of interest in a target neuron using a cell-specific promoter. The mRNA tagging strategy in C. elegans is a powerful technique that enables the identification of specific combinations of genes that define the differentiation and function of specific neurons[1-8]. In this approach, an epitope-tagged Poly(A) binding protein (3× FLAG-PAB-1) is expressed transgenetically under the control of a cell-specific promoter. $3 \times$ FLAG-PAB-1 bound transcripts are then immunoprecipitated using an anti-FLAG antibody. Gene expression profiles created by mRNA tagging have been reported for almost all major tissues from neurons to body wall muscles and intestines.

Despite the proven advantage of this technique, the conventional protocol consists of two laborious and time-consuming procedures. First, 3× FLAG-PAB-1, carried as an extrachromosomal array must be integrated into the chromosomes in order to stably express them under the control of a cell-specific promoter. This integration requires a subsequent outcross procedure using wild-type N2. Second, to prepare a large amount of synchronized animals and obtain a sufficient amount of mRNAs for subsequent quantitative PCR analysis or microarray analysis, integrant animals cultivated on six 150-mm Petri plates have previous been alkaline bleached [6]. Alternatively, obtained integrant 
animals could be grown in liquid culture[1,2]. However, these preparations appeared to be laborious and limited in practice when the mRNA tagging technique was applied to compare the differences in gene expression between animals cultivated in different environmental conditions, because C. elegans was usually cultivated on a 60-mm Petri plate for behavioral conditioning in chemotaxis, thermotaxis, and mechanosensory behavior.

In this study, we developed a simplified methodology for mRNA tagging. We have proved that mRNA tagging can be carried out using the extrachromosomal line instead of the integrant line. In addition, cell-specific mRNAs were successfully isolated from adult transgenic animals semi-synchronously grown from eggs laid by gravid adults during a time window of 3 hours. The ease of implementation of cell-specific gene expression analysis contributes to efficient interrogations of neural circuitry in terms of gene expression regulations.

\section{Material and methods}

\section{Strain preparation}

The C. elegans, wild-type N2 Bristol strain used in this study was maintained and handled using standard methods $[9,10]$. We used the wild-type N2 Bristol strain. Germline transformations were performed using co-injection mixes consisting of pKDK66 ges-1p::NLS GFP (50 ng/ $\mu \mathrm{l})$ and the gene for expressing $3 \times$ FLAG-PAB-1 under the $f l p-18$ promoter $(50 \mathrm{ng} / \mu \mathrm{l})$.

\section{Molecular biology}

PCR was conducted to amplify the 3× FLAG-PAB-1 cDNA fragment flanked by Acc65I and AgeI restriction enzyme sites from pSV15 (a gift from the Miller lab). This amplicon was subcloned into the HincII-digested pBluescript KS (-) vector (Addgene). The vector was then digested by Acc65I and 
AgeI. The resulting $3 \times$ Flag-PAB- 1 fragment was ligated into the pTAK50 vector that contains the 4.5 kb PCR-amplified flp-18 promoter. The resulting plasmid (pTAK75) vector was injected into wild-type worms to obtain the transgenic worm that carries 3× Flag-PAB-1 as an extrachromosomal array.

\section{Simplified mRNA tagging}

14 extrachromosomal array-carrying worms were deposited on each of the 10 NGM plates and cultivated at $20^{\circ} \mathrm{C}$. After 3 hours, the deposited postnatal day 0 (P0) worms were removed from each plate to segregate $F_{1}$ progeny. The $F_{1}$ progeny were synchronously grown to the adult stage and washed off the plates with the M9 buffer (22- $\mathrm{mM} \mathrm{KH}_{2} \mathrm{PO}_{4}, 34-\mathrm{mM} \mathrm{K}_{2} \mathrm{HPO}_{4}, 86-\mathrm{mM} \mathrm{NaCl}, 1-\mathrm{mM}$

$\mathrm{MgSO}_{4}$ ). The collected worms were dispensed into six 1.5-ml microtubes. The worms were then fixed in $0.5 \%$ formaldehyde in $1.0-\mathrm{ml}$ of $\mathrm{M} 9$ buffer for $20 \mathrm{~min}$ at $20^{\circ} \mathrm{C}$. The formaldehyde was inactivated by 125-mM glycine for 5 min and washed out by replacing the buffer with four changes of TBS (20-mM Tris-HCL pH7.5, 150-mM NaCl). The supernatant was removed, and the weight of the obtained worms was measured. At this point, the weight of worms should be 30-40- $\mu$ g. The worms were snap frozen in liquid nitrogen and stored for future use.

The worms were resuspended in 500- $\mu \mathrm{l}$ of homogenization buffer [50-mM HEPES pH 7.3, 150-mM NaCl, 1-mM EDTA, 10\% Glycerol, 0.5\% Igepal CA630, 0.6 mg/ml Heparin, 1-mM DTT, 0.2-mM PMSF, Complete mini (Roche), 50 U/ml RNase inhibitor (Takara)] and rapidly lysed by 30 passes on a Wheaten homogenizer. Large debris was sedimented by centrifugation at $18,000 \times g$ for 20 $\min$ at $4^{\circ} \mathrm{C}$, and the supernatant was placed on ice. Debris was subjected to one more lysis, and the two supernatants were combined. The volume of the lysate was roughly adjusted to 1-ml and incubated with 30- $\mu$ l of anti-Flag M2 affinity gel beads (Sigma Aldrich) for 2 hours at $4^{\circ} \mathrm{C}$. The affinity beads 
were sedimented by centrifugation at $400 \times \mathrm{g}$ for $1 \mathrm{~min}$ at $4^{\circ} \mathrm{C}$, and 1 -ml of lysis buffer [ $50-\mathrm{mM}$

HEPES pH 7.3, 150-mM NaCl, 1-mM EDTA, 10\% Glycerol, 0.2-mM PMSF, 0.5\% Igepal CA630, 0.6 $\mathrm{mg} / \mathrm{ml}$ Heparin] was added to the precipitated beads in the $1.5-\mathrm{ml}$ microtube. This microtube was set on the rotator, and beads were washed for 16 hours at $4^{\circ} \mathrm{C}$. Then, affinity beads were washed twice (for $30 \mathrm{~min}$ and $10 \mathrm{~min}$ ) with lysis buffer, and for 10 min with wash buffer [50-mM HEPES pH 7.3, 250-mM NaCl, 1-mM EDTA, 10\% Glycerol, 1-mM DTT, 0.5\% Igepal CA630]. The RNA-protein crosslinks were reversed by incubating the beads in $100-\mu l$ of elution buffer [ $50-\mathrm{mM}$ Tris-HCl pH 7.5, $1 \%$ SDS NaCl, 1-mM EDTA] for $30 \mathrm{~min}$ at $65^{\circ} \mathrm{C}$. This elution procedure was conducted twice, and the two supernatants containing RNA were combined. The resulting nucleic acid extracts were purified with the RNeasy minielute cleanup kit (Qiagen), in which mRNAs were eluted using $14-\mu \mathrm{l}$ of DEPC-treated water.

\section{Western blotting analysis}

The sample solution obtained in each step of the mRNA tagging procedure (Fig. 2) was mixed with 4× SDS sample buffer (Life Technologies) and 10× sample-reducing agent (Life Technologies), and this mixture was boiled at $95^{\circ} \mathrm{C}$ for $3 \mathrm{~min}$. At this point, the lysate could be flash frozen and stored for future use. Worm extracts were resolved by SDS-PAGE using NuPAGE 4\%-12\% Bis-Tris Gel (Life Technologies) and transferred to nitrocellulose membranes. The membranes were incubated with monoclonal anti-FLAG M2 peroxidase antibody produced in mice (Sigma Aldrich). This antibody was visualized using ECL Prime (GE Healthcare).

\section{Quantitative real-time PCR analysis}

Reverse transcription and quantification of the PCR products was performed by using a SuperScript III 
Platinum SYBR Green One-Step qRT-PCR kit (Life Technologies) and a StepOne Real-Time PCR System (Life Technologies). The isolated mRNAs were transcribed and amplified using primers specific to the genes, all of which were designed to include one intron in the PCR product amplified from the genomic DNA for each gene, such that the length and melting point were different from the product amplified from the cDNA.

\section{Results}

To isolate mRNAs from an interneuron of the mechanosensory neural circuit, we initially created the DNA plasmid, in which $3 \times$ FLAG-PAB-1 cDNA is fused with the flp-18 promoter that drives transgene expression mainly in AVA interneurons [11,12](Fig. 1). We then injected this plasmid into the N2 Bristol strain (Fig. 1(1)) and obtained a transgenic line expressing 3× FLAG-PAB-1 as an extrachromosomal array.

The mRNA tagging procedures were applied not only to the transgenic strain expressing $3 \times$ FLAG-PAB-1 but also to wild-type strains, which do not express PAB-1, as a negative control. To obtain synchronized worms, we performed a timed egg lay, in which eggs laid by gravid adult worms during a 3 hour time window were synchronously grown to the adult phase. As mentioned above, this procedure is an alternative to the alkaline bleaching procedure. On the first day, 14 extrachromosomal array-carrying worms (or 10 wild-type worms for a negative control experiment) were deposited on each of 10 NGM plates and cultivated at $20^{\circ} \mathrm{C}$. After 3 hours, the deposited postnatal day 0 (P0) worms were removed from each plate to segregate $F_{1}$ progeny. The $F_{1}$ progeny were grown to the adult stage and then fixed in $0.5 \%$ formaldehyde in M9 buffer for $20 \mathrm{~min}$.

The procedure after fixation is illustrated as a flow-chart in Fig. 2. Worms were collected and lysed by 30 passes on a Wheaten homogenizer. As shown in Fig. 2B (sup. 1 and sup. 2), 
immunoblotting analysis using anti-FLAG antibody revealed that the homogenization efficiently yields the $3 \times$ FLAG-PAB- 1 in the supernatant fraction. This $3 \times$ FLAG-PAB-1 complex was incubated with anti-FLAG M2 agarose beads for 2 hours. The $3 \times$ FLAG-PAB-1 bound agarose beads were washed and incubated with the elution buffer to reverse the formaldehyde crosslink. We could clearly detect the eluted 3× FLAG-PAB-1 complex by immunoblotting analysis. We finally purified the mRNA using the commercially available purification kit.

We then examined mRNAs, known to be expressed specifically in the AVA interneurons to study their enrichment by our simplified strategy compared with total RNAs. Quantitative polymerase chain reaction (q-PCR) analysis was performed against total RNA isolated from wild-type strains and mRNAs isolated from wild-type strains and transgenic strains carrying $3 \times$ FLAG-PAB- 1 as an extrachromosomal array by mRNA tagging. We used the primers for the amplification of the genes that were expressed specifically in AVA interneurons or in other neurons (Fig. 3a). The enrichment of glr-1 and rig-3, both of which are expressed in AVA neurons, and of $g c y-7$, which is expressed in ASE neurons, were calculated against acr-5, which is expressed in B-type motor neurons. This value is defined as the enrichment factor in Fig. 3b. At this point, we avoided calculation of the enrichment factor based on the amount of mRNAs of house keeping genes, taking into account the possibility that the amount of those mRNAs may be different between AVA neurons and other cells.

As shown in Fig. 3b, the enrichment factor for the glr-1 transcript in RNA isolated from wild-type strains and transgenic strains were 1.2 and 6.7, respectively. Furthermore, the enrichment factor for rig-3 in RNA isolated from wild-type and transgenic strains were 1.0 and 2.8, respectively. Thus, the enrichment factor for glr-1 and rig-3 indicated that these transcripts were enriched by our simplified mRNA tagging method. On the other hand, the enrichment factors of $g c y$-7 were not determined due to the small amount of its transcripts in RNA isolated from wild-type and 0.89 in 
transgenic strains, respectively, indicating that transcripts of $g c y-7$ were washed out by mRNA tagging. Taken together, our method could efficiently enrich transcripts in AVA interneurons.

\section{Discussion}

The advantages in simplicity for cell-specifically isolating transcripts likely contribute to cell-specific interrogation of neural circuitry in the context of gene expressions. On the other hand, this simplification, in comparison to previous studies, has the disadvantage that the total amounts of mRNA isolated by mRNA tagging cannot be measured before q-PCR analysis. In the method described in this paper, the quantity of mRNAs was insufficient for determining concentration using Nanodrop. However, given the sensitivity of the recently developed gene amplification technology, the quantification of cell-specific transcripts isolated by the mRNA tagging strategy does not necessarily require the measurement of the total amounts of RNA. We successfully amplified transcripts using the qRT-PCR kit that combines SuperScript III reverse transcriptase and Platinum Taq DNA polymerase. Furthermore, the recently developed WT-Ovation Pico System could amplify only 2 ng of RNA to produce labeled cDNA for microarray analysis $[5,13]$. Therefore, although the simplified mRNA tagging did not produce large amounts of RNA, the amount produced was sufficient for the subsequent quantitative gene expression analysis.

In previous studies, the mRNA tagging strategy had been used to compare the transcriptional profile of different cells. These analyses succeeded in identifying cell type specific genes and genes differently expressed through development and differentiation. On the other hand, although gene expression changes also occur during memory formation, the mRNA tagging strategy has not been applied to investigate the temporal expression pattern over a memory acquisition process. Interestingly, several previous studies identified the neural site of behavioral plasticity in the paradigm of 
mechanosensory habituation and thermotaxis. Indeed, it has been known that the C. elegans ortholog of the transcription factor cAMP responsive element binding protein CREB acts in the identified site to ensure memory formation[14,15]. Therefore, it may be interesting to investigate gene expression changes in those sites by cell-specific mRNA tagging before and after mechanosensory habituation training or cultivation temperature changes.

\section{Acknowledgements}

We thank the Caenorhabditis Genetic Center for sharing strains, A. Fire for sharing pPD plasmids, and

D. Miller for sharing the pSV15 plasmid. T.S. was supported by the Japan Society for the promotion of Science [Grants-in-Aid for Young Scientists (B)], Japan Science and Technology Agency under Precursory Research for Embryonic Science and Technology (PRESTO), and Mochida Memorial Foundation for Medical and Pharmaceutical Research.

\section{Author contributions}

T. Sugi conceived the project. Y. Ohtani and T. Sugi performed experiments. T. Sugi wrote the paper. 


\section{Figure legends}

Figure 1: Overview of the simplified mRNA tagging method

Step 1, germline transformations were performed to create the worms expressing $3 \times$ FLAG-PAB-1

cDNA under flp-18 promoter and GFP cDNA under ges-1 promoter as a visible selection marker. Step

2, extrachromosomal array-carrying worms were fixed in formaldehyde to crosslink the PAB-1 and

poly(A) RNA. Step 3, immunoprecipitation was conducted using anti-FLAG antibody. Step 4, crosslinks were reversed and RNA was purified.

Figure 2: Immunoprecipitation of RNA-PAB-1 complex

(a) Flow-chart of immunoprecipitation. In the step indicated by magenta letters, samples were checked by western blotting analysis.

(b) Immunoblotting analysis. Elute 1 and 2 were mixed before q-PCR analysis.

\section{Figure 3: Validation of the simplified mRNA tagging method}

(a) List of the genes whose transcripts were detected by q-PCR analysis. The expression pattern for each gene is indicated.

(b) q-PCR analysis of transcripts isolated by simplified mRNA tagging. The enrichments of $g l r-1$ and rig-3, both of which are expressed in AVA neurons, and of gcy-7, which is expressed in ASE neurons, 
were calculated against acr-5, which is expressed in B-type motor neurons. q-PCR analysis for each

gene was performed at least three times. Error bars indicate SEMs. Statistical comparisons were

performed by $t$-tests. Asterisks indicate $P<0.01$, where $P$ is the probability. ND indicates not detected.

\section{Reference}

[1] H. Kunitomo, H. Uesugi, Y. Kohara, Y. Iino, Identification of ciliated sensory neuron-expressed genes in Caenorhabditis elegans using targeted pull-down of poly(A) tails, Genome Biol. 6 (2005) R17.

[2] J. Takayama, S. Faumont, H. Kunitomo, S.R. Lockery, Y. Iino, Single-cell transcriptional analysis of taste sensory neuron pair in Caenorhabditis elegans, Nucleic Acids Research. 38 (2010) 131-142.

[3] P.J. Roy, J.M. Stuart, J. Lund, S.K. Kim, Chromosomal clustering of muscle-expressed genes in Caenorhabditis elegans, Nature. 418 (2002) 975-979.

[4] Y. Zhang, C. Ma, T. Delohery, B. Nasipak, B.C. Foat, A. Bounoutas, et al., Identification of genes expressed in C. elegans touch receptor neurons, Nature. 418 (2002) 331-335.

[5] J.D. Watson, S. Wang, S.E. Von Stetina, W.C. Spencer, S. Levy, P.J. Dexheimer, et al., Complementary RNA amplification methods enhance microarray identification of transcripts expressed in the C. elegans nervous system, BMC Genomics. 9 (2008) 84.

[6] S.E. Von Stetina, J.D. Watson, R.M. Fox, K.L. Olszewski, W.C. Spencer, P.J. Roy, et al., Cell-specific microarray profiling experiments reveal a comprehensive picture of gene expression in the C. elegans nervous system, Genome Biol. 8 (2007) R135.

[7] S.E. Von Stetina, R.M. Fox, K.L. Watkins, T.A. Starich, J.E. Shaw, D.M. Miller, UNC-4 represses CEH-12/HB9 to specify synaptic inputs to VA motor neurons in C. elegans, Genes Dev. 21 (2007) 332-346.

[8] W.C. Spencer, G. Zeller, J.D. Watson, S.R. Henz, K.L. Watkins, R.D. McWhirter, et al., A spatial and temporal map of C. elegans gene expression, Genome Research. 21 (2011) 325-341.

[9] C.C. Mello, J.M. Kramer, D. Stinchcomb, V. Ambros, Efficient gene transfer in C.elegans: extrachromosomal maintenance and integration of transforming sequences, Embo J. 10 (1991) 3959-3970. 
[10] S. Brenner, The genetics of Caenorhabditis elegans, Genetics. 77 (1974) 71-94.

[11] L.S. Nelson, M.L. Rosoff, C. Li, Disruption of a neuropeptide gene, flp-1, causes multiple behavioral defects in Caenorhabditis elegans, Science. 281 (1998) 1686-1690.

[12] C. Rogers, V. Reale, K. Kim, H. Chatwin, C. Li, P. Evans, et al., Inhibition of Caenorhabditis elegans social feeding by FMRFamide-related peptide activation of NPR-1, Nat Neurosci. 6 (2003) 1178-1185.

[13] T. Sugi, Y. Nishida, I. Mori, Regulation of behavioral plasticity by systemic temperature signaling in Caenorhabditis elegans, Nat Neurosci. (2011).

[14] Y. Nishida, T. Sugi, M. Nonomura, I. Mori, Identification of the AFD neuron as the site of action of the CREB protein in Caenorhabditis elegans thermotaxis, EMBO Reports. (2011).

[15] T.A. Timbers, C.H. Rankin, Tap withdrawal circuit interneurons require CREB for long-term habituation in Caenorhabditis elegans, Behav Neurosci. 125 (2011) 560-566. 


\section{(1) Co-injection with ges-1p::gfp}

Cell-specific promoter
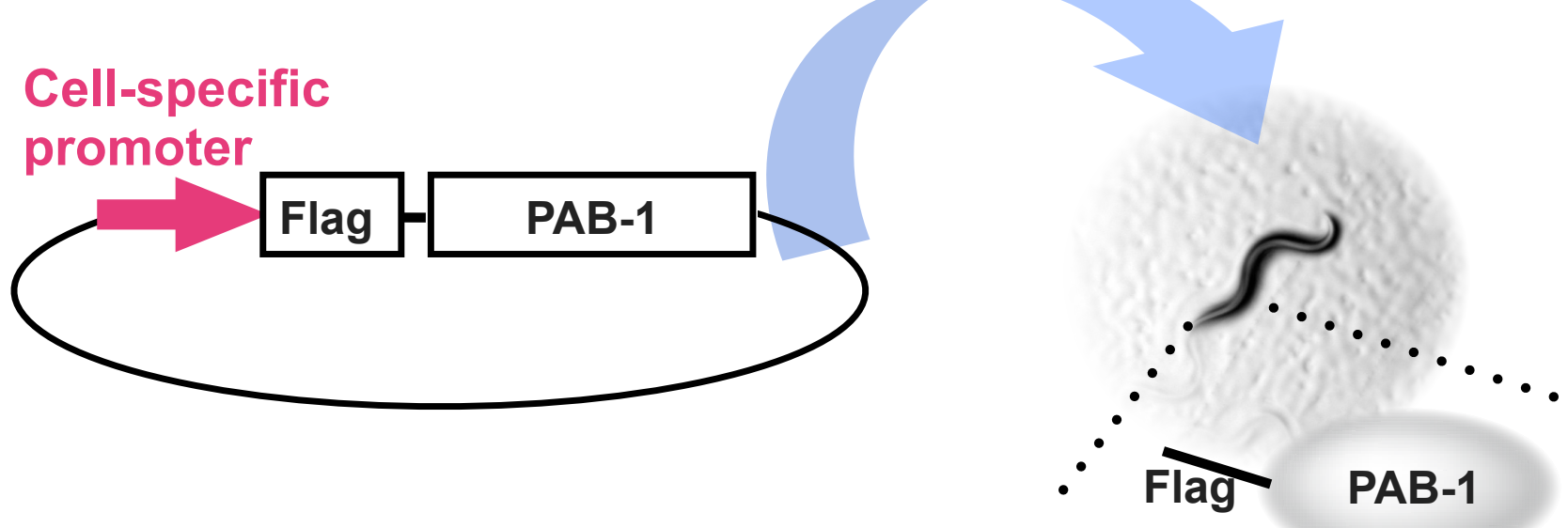

Isolation and cultivation of Ex lines expressing 3xFlag-PAB-1

(2) In vivo crosslink

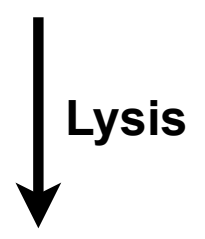

(3) Immunoprecipitation using anti-Flag antibody

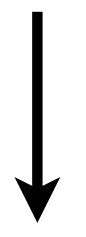

(4) mRNA isolation 
a Homogenization

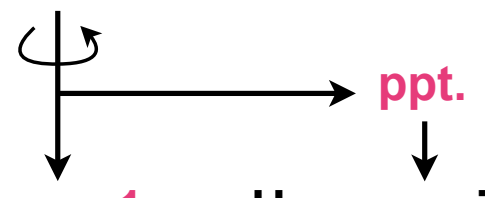

sup. 1 Homogenization

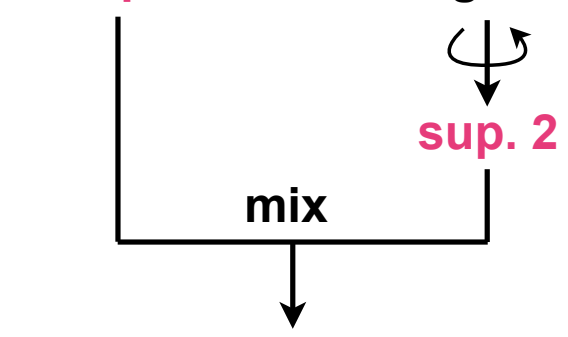

Incubation with M2 beads

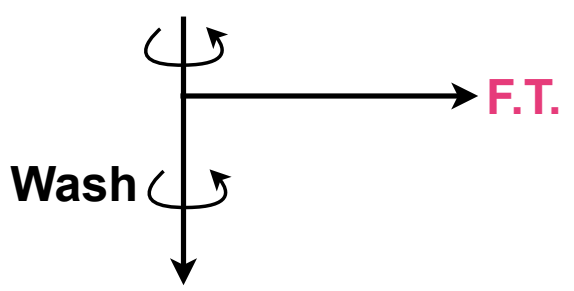

Elute $\times 2$

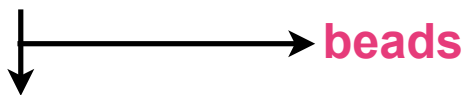

elutes 1,2

b

kDa M

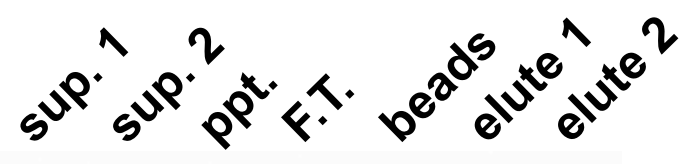

$250-$

$150-$

$100-$

75 -

$50-$

$37-$

Anti-Flag antibody 
a

\begin{tabular}{|c|c|c|}
\hline Gene & Expression pattern & Reference \\
\hline acr-5 & B-type motor neurons & $\begin{array}{l}\text { Winnier et al. } 1999 \\
\text { Kunitomo et al. } 2005\end{array}$ \\
\hline$g / r=1$ & $\begin{array}{l}\text { AVA, AVB, AVD, AVE, PVC, AIB, } \\
\text { RMD, RIM, SMD, AVG, PVQ, URY }\end{array}$ & Brockie et al. 2001 \\
\hline gcy-7 & ASE & $\begin{array}{l}\text { Yu et al. } 1997 \\
\text { Takayama et al. } 2010\end{array}$ \\
\hline rig-3 & $\begin{array}{l}\text { AVA, I1, I4, M4, NSM, inconsistent } \\
\text { in PVT }\end{array}$ & Vukojevic et al. 2012 \\
\hline
\end{tabular}

b

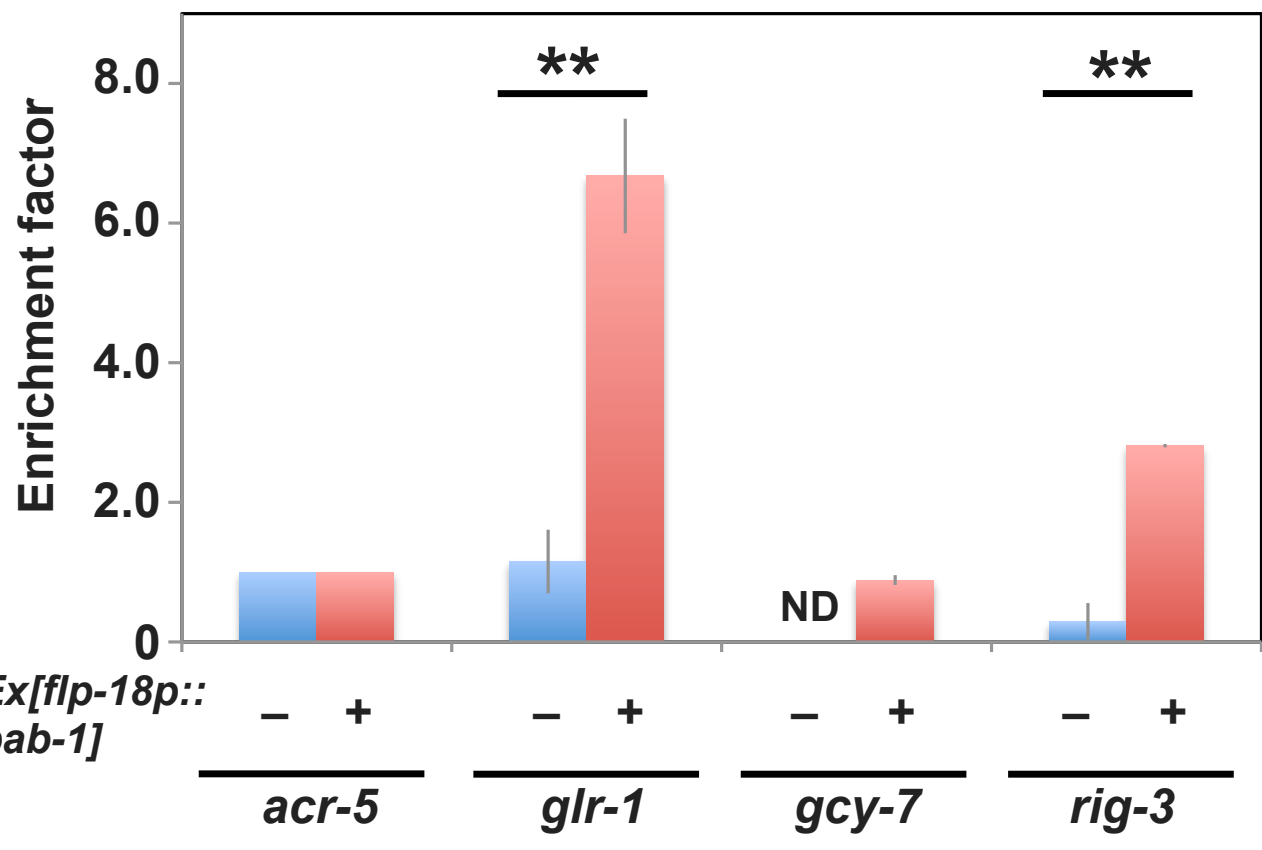

